\title{
Proto-oncoprotein Zbtb7c and SIRT1 repression: implications in high-fat diet-induced and age- dependent obesity
}

\author{
Won-II Choi ${ }^{1,2}$, Jae-Hyun Yoon', Seo-Hyun Choi ${ }^{1}$, Bu-Nam Jeon ${ }^{1}$, Hail Kim $\mathbb{B}^{2,3}$ and Man-Wook Hur $\mathbb{D}^{1}$
}

\begin{abstract}
Zbtb7c is a proto-oncoprotein that controls the cell cycle and glucose, glutamate, and lipid metabolism. Zbtb7c expression is increased in the liver and white adipose tissues of aging or high-fat diet-fed mice. Knockout or knockdown of Zbtb7c gene expression inhibits the adipocyte differentiation of 3T3-L1 cells and decreases adipose tissue mass in aging mice. We found that Zbtb7c was a potent transcriptional repressor of SIRT1 and that SIRT1 was derepressed in various tissues of Zbtb7c-KO mice. Mechanistically, Zbtb7c interacted with p53 and bound to the proximal promoter p53RE1 and p53RE2 to repress the SIRT1 gene, in which p53RE2 was particularly critical. Zbtb7c induced p53 to interact with the corepressor mSin3A-HADC1 complex at p53RE. By repressing the SIRT1 gene, Zbtb7c increased the acetylation of Pgc-1a and Ppary, which resulted in repression or activation of Pgc-1a or Ppary target genes involved in lipid metabolism. Our study provides a molecular target that can overexpress SIRT1 protein in the liver, pancreas, and adipose tissues, which can be beneficial in the treatment of diabetes, obesity, longevity, etc.
\end{abstract}

\section{Introduction}

Sirtuins (SIRT1-7) are $\mathrm{NAD}^{+}$-dependent enzymes that catalyze post-translational modifications of proteins. Sirtuins catalyze a range of deacetylation reactions, and some family members, namely SIRT2, 4, and 6 require nicotine adenine dinucleotide (NAD+) as a cofactor ${ }^{1}$. Together, these SIRTs and NAD+ regulate critical cellular pathways and are associated with aging and longevity. SIRT1 is the best-characterized member of the mammalian sirtuin family. SIRT1 deacetylases play roles in mitochondrial biogenesis, glucose and lipid metabolism, DNA repair, stress resistance, apoptosis, and inflammation ${ }^{2}$. Dysregulation of SIRT1 expression or function has significant implications in the development of obesity, diabetes,

\footnotetext{
Correspondence: Man-Wook Hur (mwhur2@yuhs.ac)

${ }^{1}$ Brain Korea FOUR Project for Medical Science, Department of Biochemistry \& Molecular Biology, Yonsei University School of Medicine, 50-1 Yonsei-Ro, SeoDaeMoon-Ku, Seoul 03722, Korea

${ }^{2}$ Graduate School of Medical Science and Engineering, Korea Advanced

Institute of Science and Technology, DaeJeon 34141, Korea

Full list of author information is available at the end of the article
}

cancer, cardiac disease, neurodegenerative disease, metabolic dysregulation, etc.

Caloric restriction, fasting, and food withdrawal increased SIRT1 expression in several rodents and human tissues, such as white adipose tissue, liver, skeletal muscle, brain, and kidney. The SIRT1 promoter contains two Hypermethylated in Cancer1 (HIC-1) response elements (HIC-1REs), an E2F1-binding element, and two p53binding elements (p53REs), insulin receptor substrate-1like binding sequences (IRS-1) and a FOXO1 forkhead-like consensus binding site (FKHD-L). SIRT1 induction is partially mediated by the interplay between cAMP response element-binding protein (CREB) and carbohydrate response element-binding protein $(\mathrm{ChREBP})^{3}$. CREB and ChREBP independently bind to the SIRT1 promoter. CREB activated by cAMP-dependent protein kinase (PKA) activates SIRT1 transcription. In contrast, ChREBP is rephosphorylated and translocated into the nucleus and represses SIRT1 transcription ${ }^{3}$. FOXO1 binds to IRS-1 and FKHD-L sites to activate rat SIRT1 transcrption ${ }^{4}$. Upon acute nutrient withdrawal, FOXO3a interacts with p53, 
and the complex binds to p53-binding sites to activate SIRT1 transcription ${ }^{5}$. p53 can be a transcription activator or repressor of the SIRT1 promoter, depending on the presence of FOXO3a. It appears that p53 plays a critical role in the transcriptional regulation of SIRT1 in response to various signals.

Interestingly, SIRT1 expression is decreased by aging and stress. An age-dependent decrease in SIRT1 expression in the liver is mediated by transcriptional repression by the C/EBP $\beta-H D A C 1$ complex ${ }^{6}$. C/EBP $\alpha$ activates SIRT1 transcription during adipogenesis by binding to the C/EBP binding element ${ }^{7}$. SIRT1 protects cells from p53and FOXO3-mediated apoptosis in response to oxidative stress $^{8,9}$. NF- $\mathrm{kB}$, which is a crucial stress response mediator, was shown to activate the SIRT1 promoter, and SIRT1, in turn, inhibits NF- $\mathrm{KB}$ function by deacetylating $\mathrm{NF}-\mathrm{\kappa B}^{10-12}$.

Interestingly, the $\mathrm{POZ}$ domain and Krüppel (POK) family of transcription factors ( $\mathrm{HIC1}, \mathrm{HIC2}$, and Zbtb7c) regulate the expression of SIRT1. HIC1 (hypermethylated in cancer 1) complexed with CtBP represses SIRT1 transcription $^{13}$. When DNA is damaged, HIC-1 and p53 jointly repress the SIRT1 gene by binding to two HIC1 REs and two p53REs, respectively ${ }^{14}$. Recently, HIC2 was shown to activate the transcription of $S I R T 1^{15}$. Zbtb7c, encoded by $Z b t b 7 c$, represses transcription by recruiting cofactor complexes (e.g., HDAC, NuRD, and BCoR complexes $)^{16-19}$. Immunohistochemical analyses of Sirt1 expression in various tissues of $Z b t b 7 c-\mathrm{KO}$ mice showed that Sirt1 is derepressed in all tissues tested (unpublished data), suggesting that Zbtb7c may be a transcriptional repressor of Sirt1.

Zbtb7c is a proto-oncoprotein that increases cell proliferation by repressing the transcription of $p 21 C d k n 1 a$ by inhibiting p53 or Miz-1 ${ }^{17,18}$. Zbtb7c increases Fasn transcription $^{20}$. Recently, we showed that Zbtb7c is vital in gluconeogenesis during fasting and in glutamine metabolism in cancer cells ${ }^{21,22}$. These results suggested that Zbtb7c might be an essential regulator of metabolism.

Despite numerous publications on Sirtuin family proteins, our understanding of these proteins and the molecular mechanism by which dietary conditions and aging regulate the SIRT1 gene is still limited, and more studies are necessary. In this study, we found that Zbtb7c plays a vital role in lipid metabolism by repressing SIRT1 transcription through a unique mechanism that involves molecular interaction between Zbtb7c and p53 and switching the p53-interacting transcription coregulators from $\mathrm{p} 300$ to $\mathrm{mSin} 3 \mathrm{~A}-\mathrm{HDAC} 1 / 3$ at p53RE2.

\section{Materials and methods \\ Mice}

Animal experiments were approved by the Committee on Animal Investigations of the Department of
Laboratory Animal Resources, Yonsei Biomedical Research Institute, Yonsei University College of Medicine. C57BL/6J mice were purchased from Charles River Japan (Yokohama, Japan). The mice were housed in a specific pathogen-free barrier facility under a 12-h light-dark cycle. Food and water were provided ad libitum. At 12 weeks of age, mice were fed a high-fat diet (HFD, 60\% fat calories) for 14 weeks. The mice were euthanized by cervical dislocation.

\section{Plasmids, antibodies, and reagents}

The pcDNA3.0-FLAG-Zbtb7c and pcDNA3.1-Zbtb7c constructs were prepared by cloning full-length cDNA into pcDNA3.0 or pcDNA3.1 (Invitrogen, CA). The pGL2-SIRT1-Luc human SIRT1 promoter-luciferase reporter gene fusion construct was generated by cloning SIRT1 promoter DNA ( $-1250 \sim+79 \mathrm{bp})$ into pGL2-Basic (Promega, WI). The pcDNA3-p53, pcDNA3-Sp1, and corepressor expression vectors have been reported elsewhere $^{4,5}$. All plasmid constructs were verified by DNA sequencing. Descriptions of the recombinant adenovirus shRNA and siRNA against Zbtb7c mRNA have been reported elsewhere ${ }^{18,21}$. Antibodies against p53, p300, SIRT1, AMPK, phosphorylated APMK, PGC-1, PPAR Zbtb7c, acetylated lysine, GAPDH, FLAG-Tag, Myc-Tag, $\mathrm{Ac}-\mathrm{H} 3$, Ac-H4, and mSin3A were purchased from Upstate (Charlottesville, VA), Chemicon (Temecula, CA), Cell Signaling Technology (Beverly, MA), Calbiochem (San Diego, CA), and Santa Cruz Biotech (Santa Cruz, CA). A rabbit polyclonal antibody against Zbtb7c was prepared in-house using recombinant GST-POZ (a.a. 1-120) and purified using Affi-Gel 10 (Bio-Rad, CA). Most of the chemical reagents were purchased from Sigma (St. Louis, MO).

\section{Cell cultures}

Various cell types were cultured in the media recom-

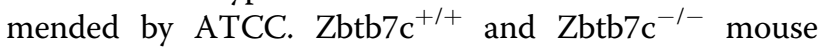
embryonic fibroblasts (MEFs) were prepared through a standard protocol and cultured in Dulbecco's Modified Eagle Medium (DMEM) (Gibco-BRL, MD) supplemented with $10 \%$ fetal bovine serum (FBS) (Gibco-BRL, MD).

\section{Serum lipid analysis}

Cholesterol, triacylglycerol, and total lipids were measured by an enzymatic colorimetric or a colorimetric method using an autoanalyzer (BS-400; MINDRAY, China).

\section{Transcription analysis of SIRT1 promoters}

The promoter-reporter fusion construct pGL2-SIRT1Luc and the expression vectors pcDNA3-FLAG-Zbtb7c, pcDNA3.1-Zbtb7c, pcDNA3-p53, pcDNA3.1-HIC-1, and pCMV-LacZ, in various combinations were transiently 
Table 1 List of oligonucleotides used in the RT-qPCR analysis of mRNA expression levels and the ChIP qPCR analysis.

\begin{tabular}{|c|c|c|}
\hline & Forward & Reverse \\
\hline \multicolumn{3}{|l|}{ Gene } \\
\hline Zbtb7c & 5'-CCCATCTGCCACAAAGTCATC-3' & 5'-TGGTGCACATGTATGGCTTCTC-3' \\
\hline Ap2 & 5'-AAGGTGAAGAGCATCATAACCCT-3' & 5'-TCACGCCTITCATAACACATTCC-3' \\
\hline $\mathrm{Cd} 36$ & 5'-AAGCTATTGCGACATGATT-3' & 5'-GATCCGAACACAGCGTAGAT-3' \\
\hline Fasn & 5'-GCTGGCATTCGTGATGGAGTCGT-3' & 5'-AGGCCACCAGTGATGATGTAACTCT-3' \\
\hline Scd1 & 5'-ACGCCGACCCTCACAATTC-3' & 5'-CAGTTTTCCGCCCTTCTCTIT-3' \\
\hline Fsp27 & 5'-GCCACGCGGTATTGCCAGGA-3' & 5'-GGGTCTCCCGGCTGGGCTTA-3' \\
\hline G6pc & 5'-AAGCCAACGTATGGATTCCG-3' & 5'-ACAGCAATGCCTTGACAAGACT-3' \\
\hline Pepck & 5'-TCTCTGATCCAGACCTTCCAA-3' & 5'-GAAGTCCAGACCGTTATGCAG-3' \\
\hline Cpt1a & 5'-GATGAACTTCTTCTTCCAGGAGTGC-3' & 5'-ATGGCAGAGGCTCACCAAGC-3' \\
\hline Cyp4a10 & 5'-AGAGTCTGGTGCAAACCTGG-3' & 5'-CCCTGATGGACGCTCTTTAC-3' \\
\hline Mcad & 5'-GATGCATCACCCTCGTGTAAC-3' & 5'-AAGCCCTITTCCCCTGAAG-3' \\
\hline Pdk4 & 5'-AGGGAGGTCGAGCTGTTCTC-3' & 5'-GGAGTGTTCACTAAGCGGTCA-3' \\
\hline Pparg & 5'-GCATGGTGCCTTCGCTGA-3' & 5'-TGGCATCTCTGTGTCAACCATG-3' \\
\hline Pgc-1 & 5'-GGCACGCAGCCCTATTCA-3' & 5'-CGACACGGAGAGTTAAAGGAAGA-3' \\
\hline Human SIRT1 & 5'-GTATTTATGCTCGCCTTGCTG-3' & 5'-TGACAGAGAGATGGCTGGAAT-3' \\
\hline Mouse SIRT1 & 5'-GATCCTTCAGTGTCATGGTT-3' & 5'-GAAGACAATCTCTGGCTTCA-3' \\
\hline $18 \mathrm{~S}$ & 5'-ATTGGAGCTGGAATTACCCGC-3' & 5'-CGGCTACCACATCCAAGGAA \\
\hline \multicolumn{3}{|l|}{ Promoter } \\
\hline Pdk4 & 5'-ATTGGCTACTGTAAAAGTCCCG-3' & 5'-TCCCAGGTCGCTAGGACTTCAG-3' \\
\hline Mcad & 5'-CCTTGCCCGAGCCTAAAC-3' & 5'-GTCTGGCTGCGCCCTCT-3' \\
\hline aP2 & 5'-AATGTCAGGCATCTGGGAAC-3' & 5'-GACAAAGGCAGAAATGCACA-3' \\
\hline $\mathrm{Cd} 36$ & 5'-ATTTGTGGTTGGTTGCCAAG-3' & 5'-AGGTGATGGGTCTTCACCAG-3' \\
\hline
\end{tabular}

transfected into cells using Lipofectamine Plus reagent (Invitrogen, CA) and analyzed as described elsewhere ${ }^{15}$.

\section{Reverse transcription and quantitative PCR (RT-qPCR) of Zbtb7c, SIRT1, and SIRT1 target mRNA}

Total RNA was isolated from the various cells using TRIzol reagent (Invitrogen, CA). cDNA was synthesized from $5 \mu \mathrm{g}$ of total RNA, random hexamers (10 pmol), and Superscript reverse transcriptase II (200 units) (Invitrogen, CA). RT-qPCR was performed using SYBR Green Master Mix (Applied Biosystems, CA) in an Applied Biosystems ${ }^{\circledR 7} 7500$ Real-Time PCR system. The oligonucleotide primers used for the qPCR assays are listed in Table 1.

\section{Western blot analysis}

Cells were harvested and lysed in RIPA buffer $(50 \mathrm{mM}$ Tris- $\mathrm{HCl}, \mathrm{pH}$ 8.0; $1 \%$ NP-40; 0.25\% sodium deoxycholic acid; $150 \mathrm{mM} \mathrm{NaCl} ; 1 \mathrm{mM}$ EGTA; and complete miniprotease cocktail). Cell extracts $(40 \mu \mathrm{g})$ were separated using $10 \%$ sodium dodecyl sulfate-polyacrylamide gel electrophoresis (SDS-PAGE), transferred onto ImmunBlot $^{\mathrm{TM}}$ polyvinylidene difluoride (PVDF) membranes (Bio-Rad, CA), and blocked with 5\% skim milk (BD Biosciences, MD). The blotted membranes were incubated with antibodies against His-tag, Myc-tag (Cell Signaling, MA), FLAG-tag (Sigma, MO), GAPDH (Chemicon, CA), p53, HIC-1, Zbtb7c, mSin3A, and SIRT1 and then incubated with either an anti-mouse or antirabbit secondary antibody conjugated to HRP (Vector Laboratory, CA). Protein bands were visualized using an ECL solution (PerkinElmer, CA).

For western blot analysis of mouse tissues, frozen tissue samples were homogenized, and tissue lysates were extracted by incubating cells in RIPA buffer $(25 \mathrm{mM}$ Tris$\mathrm{HCl}$, pH 7.6; $150 \mathrm{mM} \mathrm{NaCl} ; 1 \% \mathrm{NP}-40 ; 1 \%$ sodium deoxycholate; and $0.1 \%$ SDS) plus protease inhibitors (Roche). Supernatants were collected following a brief centrifugation, and protein concentrations in the supernatants were measured using a $\mathrm{BCA}$ protein assay kit 
(Thermo Scientific, Rockford, IL, USA). The lysates were then mixed with equal volumes of $2 \times$ Laemmli buffer ( $4 \%$ SDS, $20 \%$ glycerol, $10 \%$ 2-mercaptoethanol, $0.01 \%$ bromophenol blue, and $120 \mathrm{mM}$ Tris- $\mathrm{HCl}, \mathrm{pH} 6.8$ ) and boiled for $10 \mathrm{~min}$ at $95^{\circ} \mathrm{C}$. Next, the protein samples were separated by SDS-PAGE and transferred to a PVDF membrane (Millipore). After blocking in a 5\% skim milk solution (Sigma), the membranes were incubated with the following specific primary antibodies: anti-ZBTB7c antibody (diluted 1:1000, Biorbyt orb349355), anti-p53 antibody (diluted 1:1000, BD Pharmingen ${ }^{\mathrm{TM}}$ 554157), and anti-Actin antibody (diluted 1:1000, Cell Signaling \#3700). The membranes were then washed with $1 \times$ TBST and incubated with an anti-rabbit IgG horseradish peroxidaselinked antibody or anti-mouse IgG antibody. The detection of each protein was performed using SuperSignal West Pico Chemiluminescent Substrate (Thermo Scientific) according to the manufacturer's instructions. Signals were captured by a ChemiDoc MP system (Bio-Rad). Blots of tissue and cell lysates were quantified using ImageJ (NIH).

\section{Coimmunoprecipitation/western blot assays}

Protein extracts from liver tissue were washed, pelleted, and resuspended in lysis buffer supplemented with protease inhibitors $(20 \mathrm{~mm}$ Tris- $\mathrm{HCl}, \mathrm{pH} 7.5 ; 150 \mathrm{~mm} \mathrm{NaCl}$; $10 \%$ glycerol; $1 \%$ Triton X-100; complete mini-protease mixture (1 tablet $/ 50 \mathrm{ml}$, Roche Applied Science)). Cell lysates were precleared, and the supernatants were incubated overnight with an anti-p53 antibody on a rotating platform at $4{ }^{\circ} \mathrm{C}$ and then incubated with protein $\mathrm{A} / \mathrm{G}$ PLUS-Agarose beads (Santa Cruz, sc-2003). The beads were collected, washed, and resuspended in equal volumes of $5 \times$ SDS loading buffer. The immunoprecipitated proteins were separated using 8 and 10\% SDSPAGE. Western blot analysis was performed with the appropriate antibodies as described above.

\section{Chromatin immunoprecipitation and quantitative PCR (ChIP-qPCR) assays}

ChIP assays were performed to assess in vivo Zbtb7c binding to distinct gene promoter regions. The primers used are listed in Supplementary Table 1. NIH3T3 and MEFs were transfected with increasing amounts of Zbtb7c expression vector, pcDNA3.0-FLAG-Zbtb7c, or pcDNA3.1-Zbtb7c. By ChIP assays, the molecular interactions between Zbtb7c and p53 and histone modifications at the endogenous SIRT1 promoter were analyzed. The levels of FLAG-tagged Zbtb7c binding at the p53REs were analyzed using an anti-FLAG antibody (Sigma, Cell Signaling). The levels of Zbtb7c and p53 protein binding to the endogenous SIRT1 promoter were analyzed by ChIP using polyclonal antibodies against Zbtb7c and p53 (Santa Cruz, CA). The following oligonucleotide primer sets were designed to amplify the regions flanking the p53-binding sites within the SIRT1 promoter and acetylated histones $\mathrm{H} 3$ and $\mathrm{H} 4$ ( -278 to $-97 \mathrm{bp}$ ): forward 5'-CACTACACGCTCGCCACAAAGAGG-3', and reverse 5'-GGAGATTTAAACCCCATCACGTGACC-3' .

\section{Immunoprecipitation assays}

NIH3T3 cells, HCT116 p53 $3^{+/+}$cells, HCT116 p53-/cells, and MEFs were harvested and resuspended in lysis buffer $(20 \mathrm{mM}$ Tris- $\mathrm{HCl}, \mathrm{pH} 7.5 ; 150 \mathrm{mM} \mathrm{NaCl} ; 10 \%$ glycerol; and $1 \%$ Triton X-100) supplemented with protease inhibitors. Cell lysates were precleared, supernatants were incubated overnight with antibodies against Zbtb7c, p53, Sp1, Myc, or p300 or a corepressor on a rotating platform at $4{ }^{\circ} \mathrm{C}$ and then incubated with protein A-Sepharose Fast Flow beads. The beads were collected, washed, and resuspended in an SDS loading buffer. The immunoprecipitated proteins were separated with 10-12\% SDS-PAGE, and western blot assays were performed as described above.

\section{Oligonucleotide pull-down assays}

NIH3T3 and HEK293 cells were lysed in HKMG buffer (10 mM HEPES, pH 7.9; $100 \mathrm{mM} \mathrm{KCl;} 5 \mathrm{mM} \mathrm{MgCl}$; $10 \%$ glycerol; $1 \mathrm{mM}$ DTT; and 0.5\% NP40). Cell extracts were incubated with $1 \mu \mathrm{g}$ of biotinylated double-stranded oligonucleotides (p53RE1 and p53RE2) for $16 \mathrm{hr}$. The oligonucleotide sequences were as follows (only the top strands are shown): p53RE1, 5'-AACAGCCTCCGCCCG CCACGTGACCCGTAGTGTT-3', and p53RE2, 5'-TGT TGTGGTCTGGCCCGCGTGGGTGGCGGGAGCG-3'). The core p53-binding sequences are underlined. The core CGTG sequence was mutated in the pGL2-SIRT1-Luc reporter mutant plasmids (Fig. 3b). The mixtures of cell lysate and biotinylated oligonucleotide were incubated with streptavidin-agarose beads for $2 \mathrm{~h}$, washed with HKMG buffer, and precipitated by centrifugation. The precipitates were analyzed by western blotting using antibodies against Zbtb7c, p53, and GAPDH, as described above.

\section{Statistical analysis}

Student's $t$ test was used for statistical analyses.

\section{Results}

Zbtb7c regulates the transcription of SIRT1, Ppary, and Pgc$1 a$ and various genes encoding lipid metabolism enzymes

Previously, we found that Zbtb7c regulates the expression of fatty acid synthase, a key enzyme of fatty acid synthesis, suggesting that Zbtb7c plays a vital role in lipid metabolism ${ }^{20}$. Interestingly, in the present study, abdominal adipose tissue was significantly decreased in aging Z Zbtb7c-KO mice (Fig. 1a, b). Histological sections of gonadal WAT from the $Z b t b 7 c-\mathrm{KO}$ mice showed 


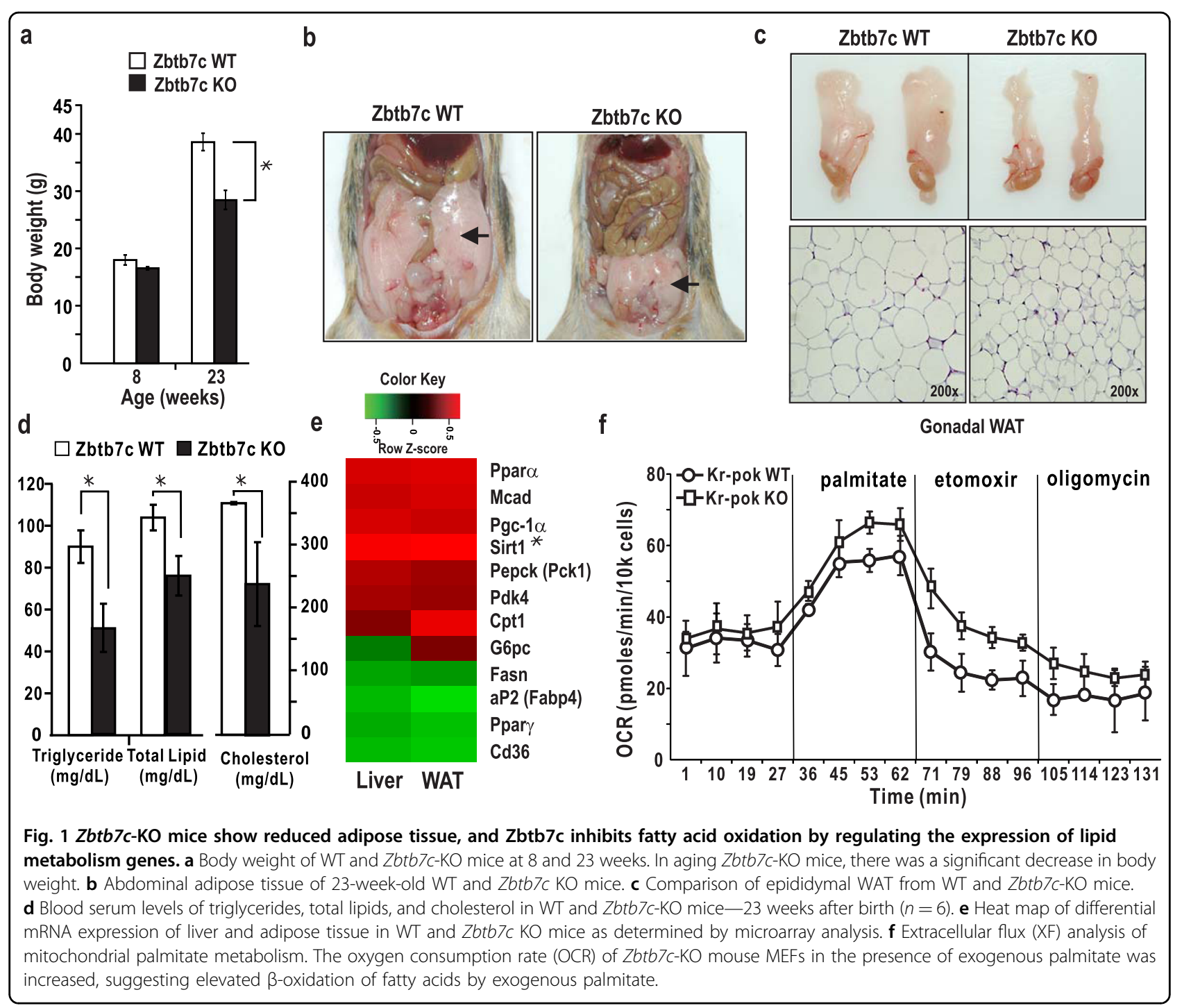

adipocytes that were smaller than those in the control mice (Fig. 1c). We also found significant decreases in triglycerides, total lipids, and serum cholesterol in the blood of the Zbtb7c-KO mice (Fig. 1d).

Microarray analyses of differentially expressed genes in the liver and WAT tissues of the Zbtb7c-KO mice showed changes in the expression of genes important in lipid metabolism (Fig. 1e and Supplementary Fig. 1). In Zbtb7cKO mice, the mRNA expression of two critical metabolic regulators, Ppary and Pgc-1 $\alpha$, and several other genes involved in fatty acid metabolism $(A p 2, S c d 1, C d 36$, and Fans), adipocyte differentiation (Ppary), and gluconeogenesis (G6pc and Pepck) were decreased. In contrast, the expression of genes involved in fatty acid $\beta$-oxidation or mitochondrial biogenesis (Cpt1a, Cyp4a10, Mcad, and $P g c-1 \alpha)$ was increased. In particular, in both liver and WAT tissues, the expression of Ppary was downregulated, and that of $P g c-1 \alpha$ was significantly upregulated.
Moreover, the expression of SIRT1, an essential regulator of glucose production in the liver (via TORC2, PGC- $1 \alpha$, and FOXO1), and lipid mobilization and metabolism in adipose tissue (through PPAR $\gamma$ and LXR) were significantly increased in Zbtb7c-KO mice (Supplementary Fig. 1).

Using western blot assays, we also analyzed the expression levels of signaling molecules upstream of Pgc$1 \alpha$ and Ppary, such as Ampk, phosphorylated Ampk, Akt, and phosphorylated Akt in the liver and WAT. While the total expression of Ampk remained unchanged, that of phosphorylated Ampk, an activated form of Ampk that stimulates $\beta$-oxidation and ketogenesis and inhibits fat synthesis $^{23}$, was significantly increased in the $Z b t b 7 c-K O$ mice (Supplemental Fig. 1b, d). The expression of $P g c-1 \alpha$ was also upregulated, as expected. We also analyzed the expression of Akt and phosphorylated Akt in WAT tissues. Total Akt expression was similar in the WT and 
knockout mice. Nevertheless, the expression of phosphorylated Akt, a multifunctional kinase that plays critical roles in the inhibition of lipolysis and synthesis of fatty acids $^{23-25}$, was significantly decreased by Zbtb7c knockout. These results suggest that Zbtb7c plays an essential role in lipid metabolism by regulating the expression and activity of upstream signaling molecules such as Ampk and Akt and the transcription of SIRT1 and Ppary, $P g c-1 \alpha$, genes encoding lipid metabolism regulators in the liver and WAT.

Because the expression of the genes involved in fatty acid $\beta$-oxidation was increased in $Z b t b 7 c-\mathrm{KO}$ mice, we investigated the oxygen consumption rates (OCRs) of Zbtb7c WT and KO MEFs using a Seahorse XF24 Flux Analyzer. $Z b t b 7 c-K O$ MEFs treated with palmitate had a higher baseline OCR. The Zbtb7c-KO MEFs showed an increase in the palmitate-induced OCR, indicating higher activity of fatty acid oxidation than induced in the WT Zbtb7c MEFs (Fig. 1f).

\section{Zbtb7c downregulates the transcription of SIRT1 and stimulates adipocyte differentiation}

The expression of $P g c-1 \alpha$ was upregulated and that of Ppary was downregulated in the Zbtb7c-KO mice. SIRT1 expression was derepressed in the liver and WAT of the $Z b t b 7 c-K O$ mice. These changes were likely to increase fatty acid oxidation and reduce fat mass. Considering that the $P g c-1 \alpha$ and Ppary genes are downstream of SIRT1 and that a negative correlation was found between SIRT1 and Zbtb7c expression, we investigated whether Zbtb7c can repress human SIRT1 transcription and thereby regulate Pgc- $1 \alpha$ and Ppary expression or activity in three different mouse cell types: MEFs, NIH3T3 cells, and differentiating NIH3T3-L1 cells. In MEFs with the Zbtb7c gene knocked out, SIRT1 expression was higher than that in WT MEFs (Fig. 2a, b). While ectopic Zbtb7c repressed endogenous Sirt1 expression in NIH3T3 cells, knockdown of Zbtb7c expression by infection with recombinant adenovirus expressing shZbtb7c RNA increased endogenous Sirt1 expression (Fig. 2c, d). We observed similar results in NIH3T3-L1 cells treated with adipocyte differentiation inducers.

Because SIRT1 inhibits adipocyte differentiation, we tested whether Zbtb7c can promote adipocyte differentiation by repressing SIRT1 expression. Knockdown of Zbtb7c expression derepressed or increased Sirt1 mRNA expression by 3.7-fold and inhibited adipocyte differentiation (Fig. 2e, f right). Knockdown of SIRT1 expression enhanced adipocyte differentiation (Fig. 2e). Knockdown of both Zbtb7c and SIRT1 resulted in SIRT1 mRNA expression and adipocyte differentiation similar to those of the control cells, suggesting that Zbtb7c represses SIRT1 expression and thereby affects adipocyte differentiation (Fig. 2e, f).

\section{Zbtb7c represses SIRT1 transcription}

Because SIRT1 and Zbtb7c expression showed a negative correlation, we investigated whether Zbtb7c can repress the transcription of the human SIRT1 promoter. By performing transient transfection and subsequent transcription assays of four promoter and reporter gene fusion constructs (pGL2-SIRT1-Luc) $(-1.4,-0.9,-0.6$, and $-0.3 \mathrm{~kb}$ sequences $5^{\prime}$-upstream from translation start site) in HEK293 cells, we investigated whether HIC1, p53, and Zbtb7c regulate SIRT1 transcription. HIC1 repressed expression of the SIRT1 promoter by $50-60 \%$ (Fig. 3a lanes 2, 6, 10, and 14). Zbtb7c also repressed reporter expression by $55-65 \%$ (Fig. 3a lanes 4, 8, 12, and 16). p53 potently repressed the transcription of pGL2-SIRT1-Luc, by more than $85 \%$ (Fig. 3a lanes 37,11 , and 15). We noticed that transcriptional repression by Zbtb7c was slightly more intense as the $5^{\prime}$-upstream sequence length was shortened, and transcriptional repression by p53 was somewhat weakened with the shortening of the sequences. Nonetheless, the data suggested that the proximal promoter region (bp, $-300 \sim+79$ ) was sufficient for transcriptional repression by p53 and/or Zbtb7c (Fig. 3a). Interestingly, the short SIRT1 proximal promoter region contains two p53REs (p53RE1 and p53RE2, which are $-188 \sim-181$ bp and -159 to $\sim-152$ bp from the ATG translation start codon, respectively). We meticulously investigated how the SIRT1 promoter was regulated by HIC-1, p53, or Zbtb7c or by their combination. Zbtb7c enhanced transcriptional repression of pGL2-SIRT1-Luc WT through p53 (Fig. 3b lanes 3,6), and this construct was further repressed by HIC1 (Fig. 3b lanes 3, 6, 8).

To investigate the function of p53REs in transcriptional regulation by the aforementioned factors (Zbtb7c, p53, and HIC1), we introduced mutations at p53REs by site-directed mutagenesis. The core CGTG sequences of either p53RE1 $(-188 \sim-181$ bp from the ATG translation start codon; $5^{\prime}$-ACGTGACC-3') and/or p53RE2 (-159 -152 bp in the core sequence $5^{\prime}$-GCGTGGGT-3') were replaced with AGAG. Mutations in p53RE1 and/or p53RE2 profoundly affected the transcriptional regulation of SIRT1 by p53, Zbtb7c, and HIC1 (Fig. 3b). The mutation of p53RE1 abolished transcriptional repression that had been induced by Zbtb7c, and interestingly, the mutation of p53RE2 resulted in potent transcriptional activation (2.5-fold) of the SIRT1 promoter by Zbtb7c. The results suggested that both p53RE1 and p53RE2 were involved in transcriptional repression by p53 and Zbtb7c and suggested that p53RE2 was more critical in transcriptional repression by Zbtb7c. In the mutant constructs, transcriptional repression enhancement of the promoter by coexpressed p53 and Zbtb7c was also lost and attenuated transcription repression that had been induced by p53 approximately one fold (Fig. 3b, lanes 11 and 14 for the p53RE mutant 1 and lanes 19 and 22 for the p53RE mutant). 
a

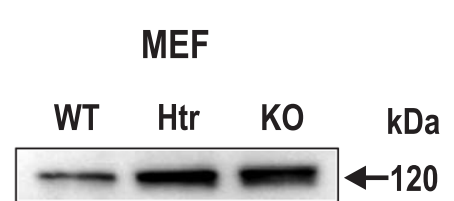

WB : anti-Sirt1

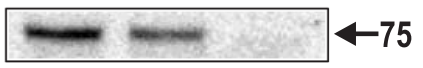

WB : anti-Zbtb7c

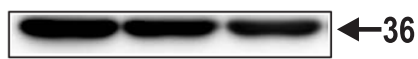

WB : anti-Gapdh

C

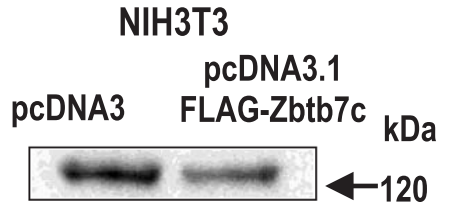

WB: anti-Sirt1

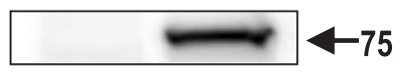

WB: anti-FLAG

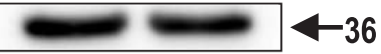

WB:anti-Gapdh

e

3T3-L1
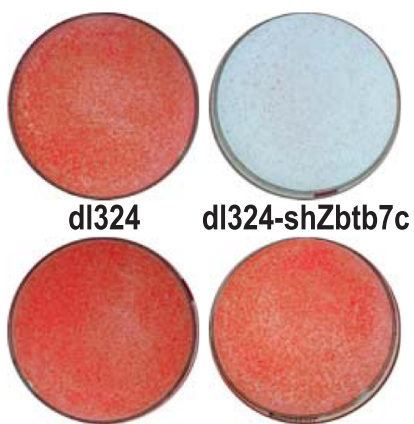

si Sirt1 RNA
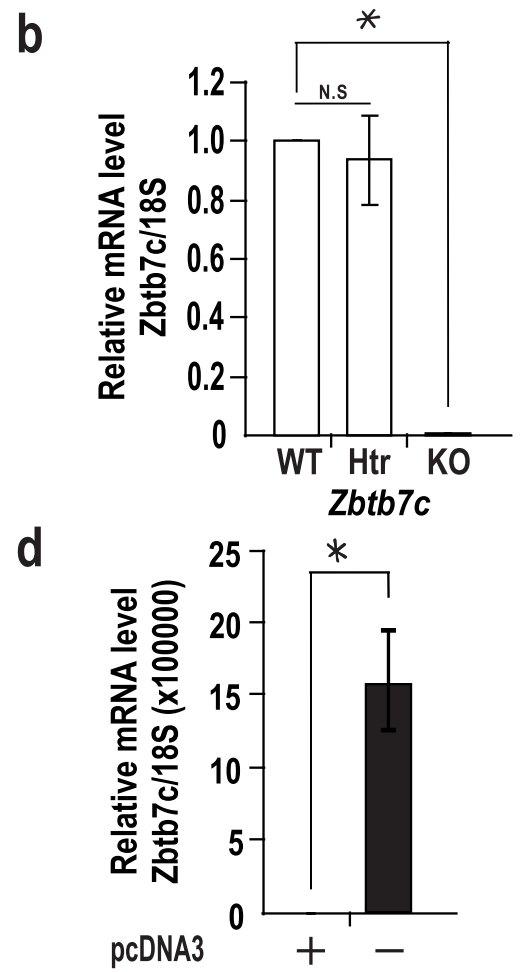

\section{f} pcDNA3.1 Zbtb7c - +

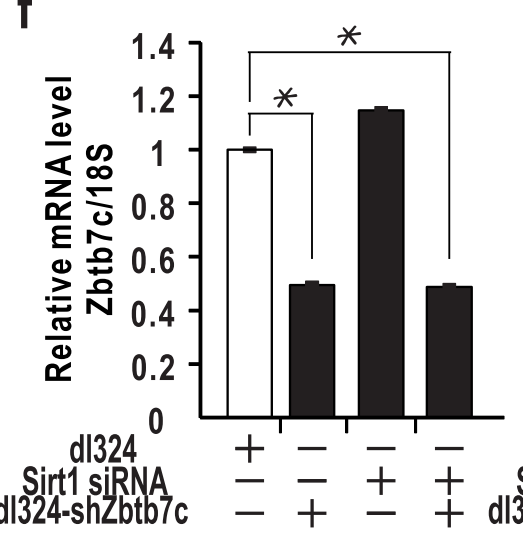

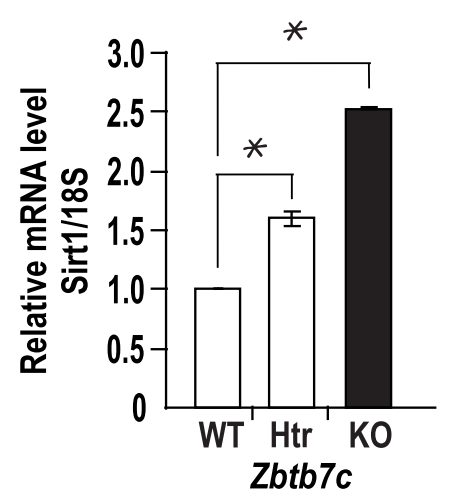
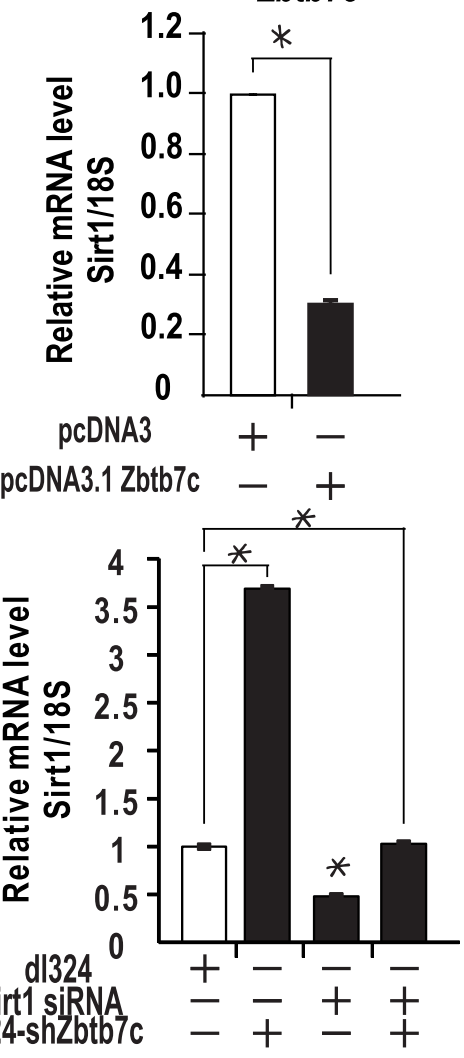

Fig. 2 Zbtb7c represses SIRT1 transcription and induces adipocyte differentiation. $\mathbf{a}, \mathbf{b}$ qRT-PCR and western blot analyses of SIRT1 and Zbtb7c expression in WT and Zbtb7c-KO MEFs. c, d qRT-PCR and western blot analyses of SIRT1 and Zbtb7c expression in NIH3T3 cells transfected with the Zbtb7c expression vector. e Zbtb7c promoted adipocyte differentiation and/or lipid synthesis via SIRT1 repression. 3T3-L1 preadipocytes were infected or transfected with recombinant Zbtb7c shRNA adenovirus and/or SIRT1 siRNA, cultured in differentiation medium for 14 days, and stained with oil red $O$ reagent. $\mathbf{f}$ qRT-PCR analysis of SIRT1 and Zbtb7c expression in 3T3-L1 cells treated as in (e).

Mutation of p53RE1 and/or p53RE2 in the SIRT1 promoter resulted in weaker transcriptional repression by $\mathrm{p} 53$ than observed for the WT promoter. The mutation of p53RE2 resulted in greater derepression. These data suggested the importance and nature of p53 binding at p53RE1 and p53RE2. p53 binding to both p53RE1 and p53RE2 was necessary and equally important in the transcriptional repression by $\mathrm{p} 53$. These data also suggested that p53RE1 was critical in the transcriptional repression by Zbtb7c, and mutation of the site abolished repression by Zbtb7c. p53RE2 was also crucial in transcriptional repression by p53 and in intense transcriptional repression by molecular interaction with Zbtb7c. Lack of p53 binding due to mutation created a situation in which the SIRT1 promoter was potently activated by Zbtb7c. In the mutant reporter constructs, coexpression of p53 and Zbtb7c did not lead to enhanced transcriptional repression as observed for the WT promoter (Fig. 3b). 
a

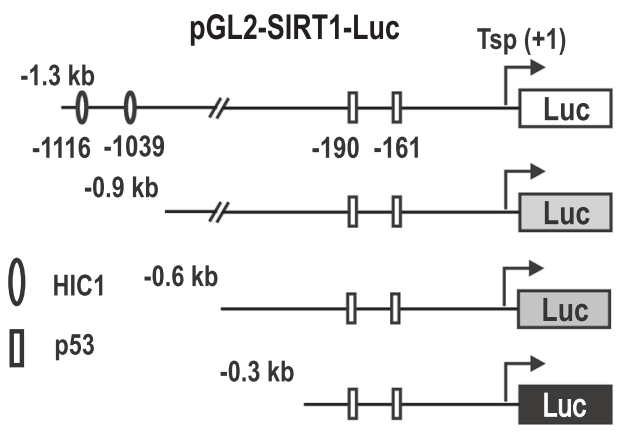

b
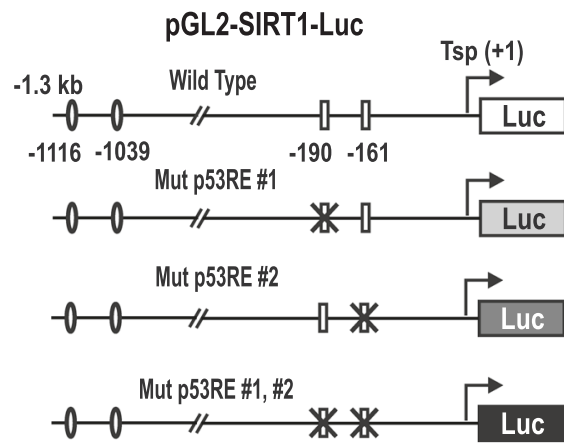

0 HIC1

\ $\mathrm{p} 53$
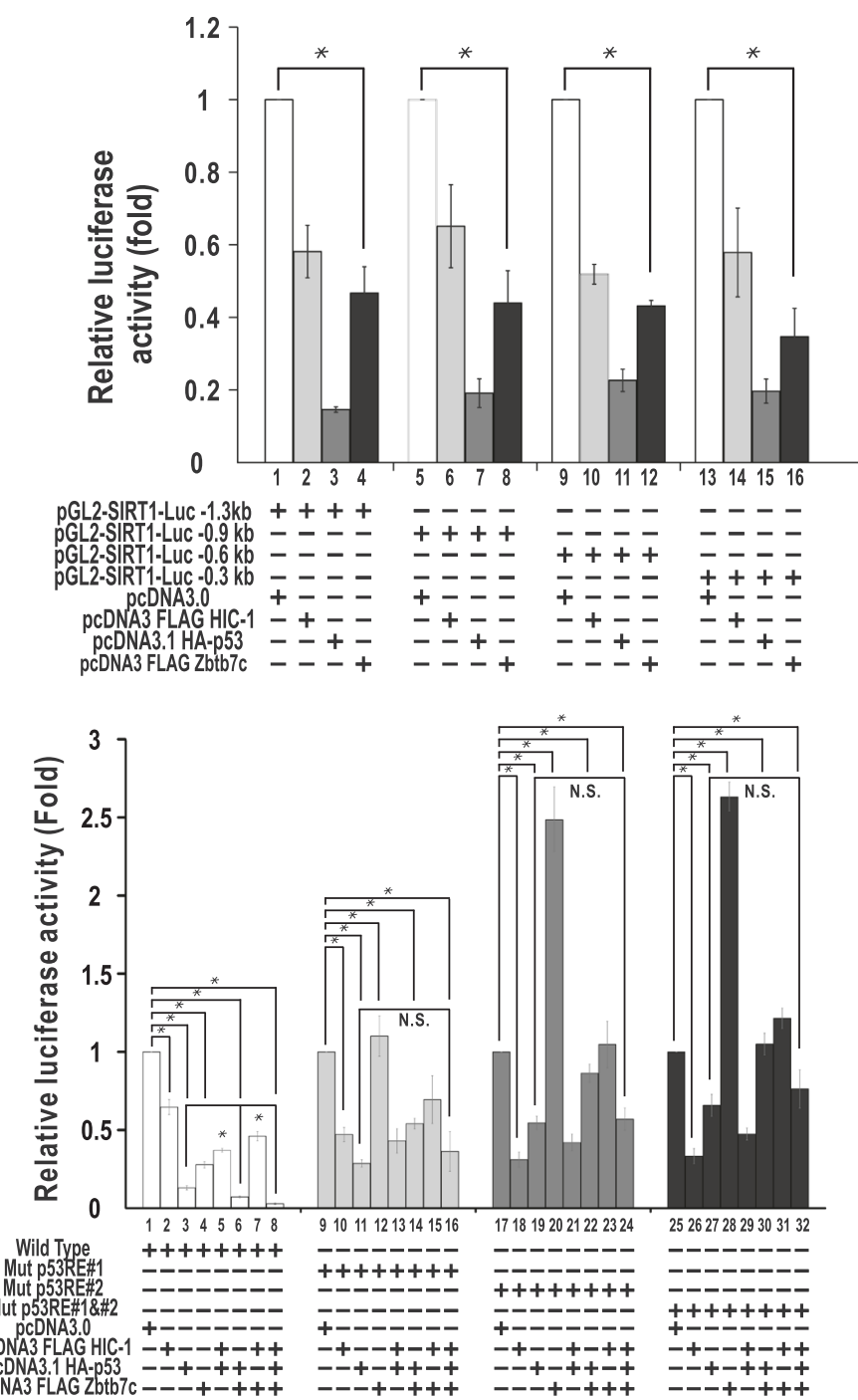

C

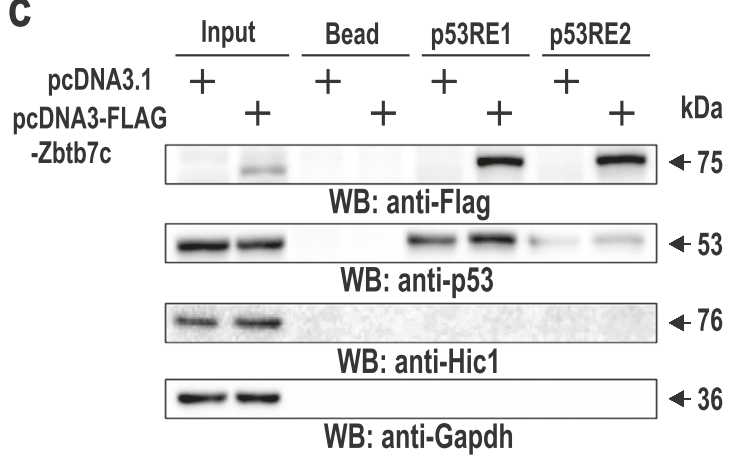

d

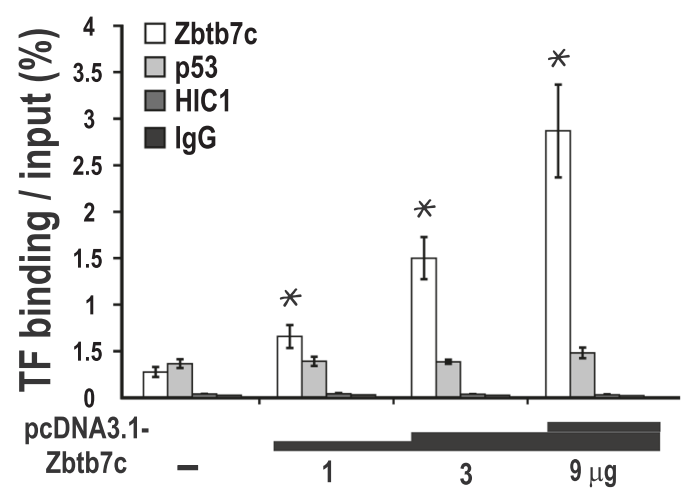

Fig. 3 Zbtb7c represses SIRT1 transcription by binding to p53REs. a Zbtb7c represses SIRT1 transcription. Transient transcription assays of the four pGL2-SIRT1-Luc reporter construct in HEK293A cells. The Zbtb7c expression vector and reporter construct were transiently cotransfected, and luciferase activities were measured. Averages of three independents assays are shown. Error bars, standard deviations. b Site-directed mutagenesis of p53RE1 and/or p53RE2 in pGL2-SIRT1-Luc-1.3 kb. Transient transfection and transcription assays were performed to identify the critical elements in transcriptional repression of the SIRT1 promoter by Zbtb7c. p53RE2 was critical to transcriptional repression by Zbtb7c. c Oligonucleotide pull-down assays of Zbtb7c binding to the p53REs of the SIRT1 promoter in NIH3T3 cells. The pulled-down proteins were then analyzed by western blotting using the antibodies indicated. Zbtb7c bound to p53REs (equally well to both elements) and increased p53 binding to these elements. $\mathbf{d}$ qChIP assays of Zbtb7c, endogenous Hic-1, p53, and ectopic Zbtb7c binding to the p53REs of the SIRT1 promoter in NHH3T3 cells. On p53RE regions, Zbtb7c binding was increased in a dose-dependent manner, but p53 binding remained constant. The antibodies used were anti-FLAG (for FLAG-tagged Zbtb7c), anti-p53, anti-Hic-1, or control IgG antibodies. Error bars, standard deviations. 


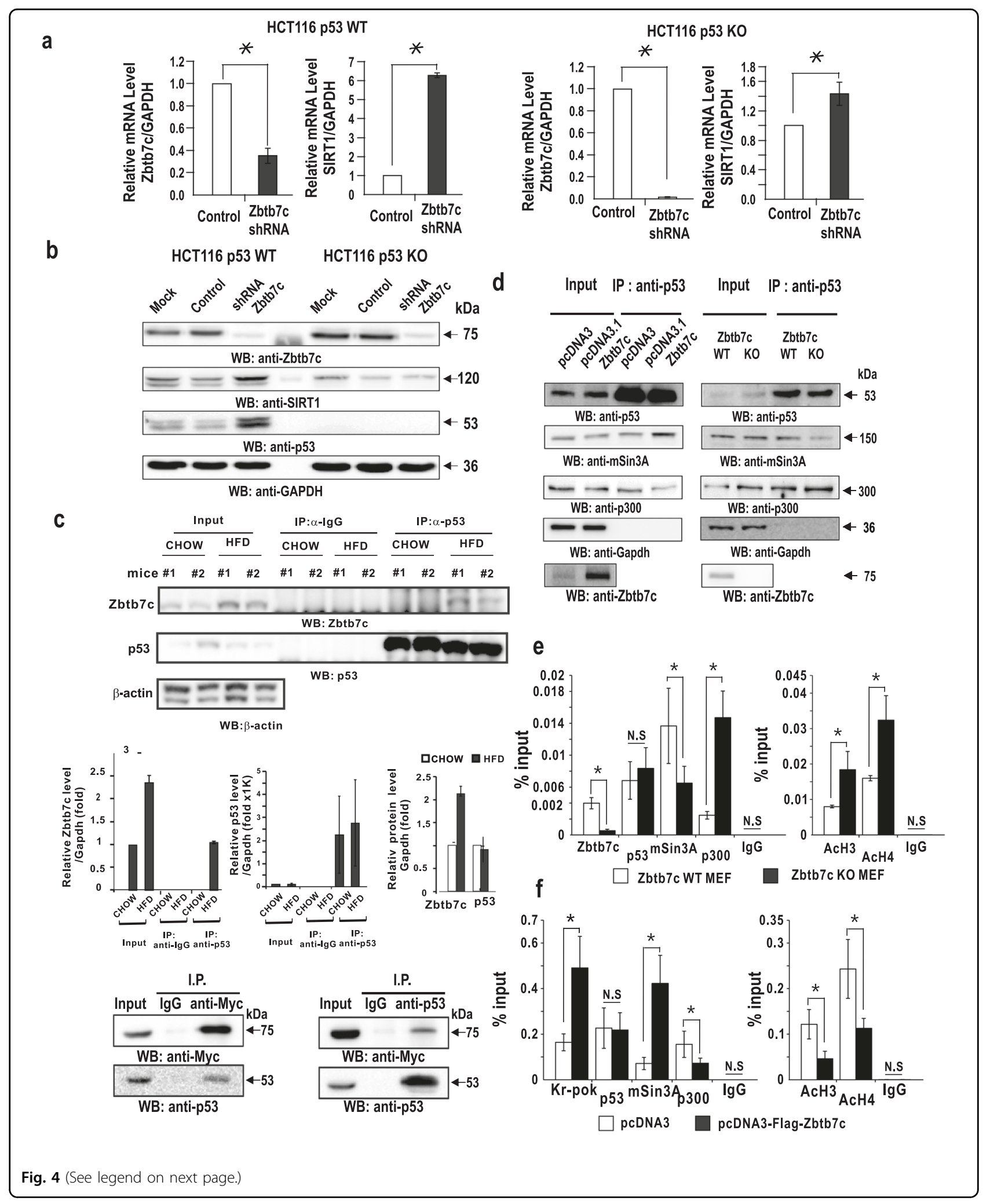


(see figure on previous page)

Fig. 4 Transcriptional repression of SIRT1 by Zbtb7c requires p53, and Zbtb7c increases binding of the mSin3A-HDAC1 complex to p53REs. a, b Zbtb7c represses SIRT1 more strongly in WT p53 HCT116 cells than in p53-KO HCT116 cells. qRT-PCR and western blot analyses of Zbtb7c and SIRT1 expression in WT TP53 and TP53-KO HCT116 cells. Cells infected with recombinant control adenovirus dl324 or recombinant dl324Zbtb7c-shRNA. GAPDH, control. c Top. p53 and Zbtb7c interacted with each other. Coimmunoprecipitation of p53 and Zbtb7c and western blot assays. Liver extracts prepared from mice fed chow or HFD and NIH3T3 cell lysates with ectopic myc-Zbtb7c expression were subjected to coimmunoprecipitation with anti-Zbtb7c or anti-p53 antibody and to western blotting for detection of the interaction with p53 or Zbtb7c, respectively. Middle, histograms of the average protein band intensities of Zbtb7c, p53, and Gapdh. Western blot images were analyzed by ImageJ. Bottom, co-IP and western blot analyses of molecular interactions between ectopically expressed Myc-tagged Zbtb7c and endogenous p53. d Coimmunoprecipitation of p53, mSin3A, and p300 and western blot assays. Cell lysates were prepared from NIH3T3 cells transfected with ectopically expressed Zbtb7c. In addition, total cellular extracts of WT and Zbtb7c-KO MEFs were prepared. The cell lysates were coimmunoprecipitated with anti-p53 antibody and subjected to western blot assay for determination of the interaction with mSin3A and p300. The precipitates were analyzed by western blotting with the antibodies indicated. GAPDH, control. e ChIP-qPCR assays were performed to assess endogenous Zbtb7c, p53, mSin3a, and p300 binding to p53REs at the SIRT1 promoter in wild-type and Zbtb7c-KO MEFs. f ChIP-qPCR assays were performed to assess endogenous Zbtb7c, p53, mSin3a, and p300 binding to p53REs at the SIRT1 promoter in NIH3T3 cells transfected with FLAGZbtb7c. Error bars, standard deviations. In addition, the acetylation status of histone H3 and H4 near the proximal SIRT1 promoter is shown. IgG, control ChIP antibody. Error bars, standard deviations.

Intrigued by these observations, we investigated the molecular events (or DNA binding) among p53, Zbtb7c, and p53REs to understand how SIRT1 gene transcription is regulated. Oligonucleotide pull-down assays also showed that ectopic Zbtb7c bound to both p53RE1 and p53RE2 with similar binding affinity. p53 bound more strongly to p53RE1 than to p53RE2, and ectopic Zbtb7c showed slightly increased p53 binding (Fig. 3c). These two different sets of data match closely, especially considering the nature of ChIP assays. The two p53REs are located very close to each other, and accordingly, primer sets amplified the DNA regions encompassing the p53RE1 and p53RE2 regions. p53RE1 appears to be the primary site for p53 binding, and the p53-Zbtb7c interaction seems to be important in Zbtb7c binding in the region (Fig. 3c)

ChIP assays of NIH3T3 cells transfected with increasing amounts of Zbtb7c expression vector showed a dosedependent increase in Zbtb7c binding to the elements spanning the two p53REs, but p53 binding remained virtually unchanged, although p53 binding after $9 \mu \mathrm{g}$ of Zbtb7c expression vector was transfected showed a weak increase. HIC1 showed little binding activity (Fig. 3d). The two different DNA-binding assays gave virtually identical results.

Because p53 is a critical regulator of SIRT1 expression in response to various stresses, Zbtb7c may repress SIRT1 transcription by modulating molecular interactions involving p53 at p53REs. We first tested whether endogenous SIRT1 transcription repression by Zbtb7c is affected by the presence or absence of p53. The knockdown of Zbtb7c by shRNA resulted in strong derepression (an approximately 6 -fold increase) of SIRT1 mRNA in WT p53-expressing HCT116 cells. However, knockdown of Zbtb7c in p53-null HCT116 cells resulted in only weak derepression (1.4-fold) of SIRT1 mRNA, suggesting that transcriptional repression by Zbtb7c strongly requires p53 activity (Fig. 4a). Western blot analyses of HCT116 cells showed similar results (Fig. $4 \mathrm{~b})$. These results indicated that Zbtb7c could potently repress SIRT1 gene transcription in cells expressing p53 and weakly repress SIRT1 gene transcription in p53-null cells. p53 appeared to play a pivotal role in the repression of SIRT1 by Zbtb7c.

\section{Zbtb7c interacts with p53, which increases p53 interaction with corepressor mSin3A-HDAC complex, not coactivator p300, decreasing the acetylation rate of histone $\mathrm{H} 3$ and $\mathrm{H} 4$ at the p53REs in the SIRT1 proximal promoter}

Interestingly, Zbtb7c more potently repressed the SIRT1 gene promoter in cells expressing p53 than in cells not expressing p53. p53 is a transcriptional repressor of the SIRT1 gene promoter. We suspected that Zbtb7c might have interacted with p53 and that this interaction led to more potent transcriptional repression of SIRT1. Co-IP and western blotting assays of the liver tissue extracted from mice fed an HFD and the cellular extracts prepared from cells transfected with the Myc-Zbtb7c and p53 expression vectors showed that the endogenous and/or ectopically expressed Zbtb7c and p53 proteins interacted with each other (Fig. 4c, top and bottom, and d).

We further investigated how Zbtb7c enhanced the transcriptional repression of the SIRT1 promoter by $\mathrm{p} 53$. p53 was previously shown to repress $p 21 C D K N 1 A$ transcription by interacting with the mSin3A-HDAC complex $^{18}$. Interestingly, our co-IP assays showed that ectopic Zbtb7c increased the interaction between p53 and the mSin3A-HDAC complex but decreased the p53 interaction with p300 (Fig. 4d, left). the Co-IP assays of Zbtb7cKO MEF extract performed with anti-p53 antibody also showed that the interaction of p53 with p300 was increased, and the interaction with the mSin3A-HDAC corepressor complex was weakened in the absence of Zbtb7c expression (Fig. 4d right). 
ChIP assays of the binding of various factors (Zbtb7c, p53, mSin3A, and p300) in Zbtb7c-KO and WT MEFs indicated that, in the absence of Zbtb7c, the binding of mSin3A was decreased, and the binding of p300 was significantly increased. The binding of p53 was not affected (Fig. 4e). In addition, the ChIP assays of NIH3T3 cells transfected with the Zbtb7c expression vector showed that while ectopic Zbtb7c expression did not affect $\mathrm{p} 53$ binding, it increased $\mathrm{mSin} 3 \mathrm{~A}$ binding to $\mathrm{p} 53 \mathrm{RE}$ sites and decreased p300 binding (Fig. 4f). These molecular changes induced by Zbtb7c decreased the acetylation of histones $\mathrm{H} 3$ and $\mathrm{H} 4$ around the p53REs of the SIRT1 promoter, which decreased SIRT1 transcription (Fig. 4e, f, right). Overall, these data suggested that Zbtb7c interacted with p53, bound to p53RE sites, and increased recruitment of the mSin3A-HDAC corepressor complex to potently repress SIRT1 transcription.

\section{Zbtb7c increases acetylation of the transcription factors} Ppary and Pgc-1a by repressing SIRT1 expression and decreasing the binding of Ppary or Pgc-1a to target genes

Most reports support the idea that SIRT1 stimulates lipid metabolism. For example, SIRT1 was previously shown to regulate adipocyte differentiation and fatty acid oxidation by deacetylating lysine residues in Ppary and Pgc- $1 \alpha^{26,27}$. Because Zbtb7c repressed SIRT1 expression, we examined whether Zbtb7c can increase the posttranslational acetylation of Ppary or Pgc-1 $\alpha$. In NIH3T3 cells overexpressing Zbtb7c, lysine acetylation of Ppary and Pgc-1 $\alpha$ was increased, and lysine acetylation of Ppar $\gamma$ and Pgc- $1 \alpha$ was decreased in the Zbtb7c-KO MEFs (Fig. 5a, b). These results indicated that Zbtb7c could have increased the acetylation of Ppary or Pgc-1 $\alpha$ through the repression of SIRT1.

Furthermore, we tested whether Zbtb7c affected the transcription of the pGL3-3x(PPRE)-TK-Luc reporter plasmid carrying three Ppary response elements in NIH3T3 cells. Transient transfection and reporter expression assays of the reporter plasmid (3xPPRE-TkLuc) showed that Ppary or Zbtb7c activated transcription and that coexpression of Ppary and Zbtb7c further increased transcription (Fig. 5c). The ChIP assays of Ppary binding to the endogenous promoters of Ppary target genes, including $a P 2$ and $C d 36$, showed an apparent decrease in Zbtb7c KO MEFs by Ppary deacetylation by derepressed SIRT1. The ChIP assays also showed decreased acetylation of histones $\mathrm{H} 3$ and $\mathrm{H} 4$ at the Ppary target gene promoters in $Z b t b 7 c-K O$ MEFs, probably due to decreased Ppary binding. This outcome may explain the low level of $a P 2$ and $C d 36$ mRNA expression in $Z b t b 7 c-K O$ MEFs and mouse liver and WAT tissues (Fig. $6 a, b$ and Supplementary Fig. 1).

We also tested whether Zbtb7c affected the transcription of endogenous Pgc- $1 \alpha$ target genes. Pgc- $1 \alpha$ weakly repressed transcription of the reporter construct pGL3-3x(PPRE)-
TK-Luc. Zbtb7c activated the reporter construct, probably by increasing lysine acetylation of Pgc-1 $\alpha$, via SIRT1 gene repression (Fig. 5c right). The ChIP assays showed increased Pgc-1 $\alpha$ binding at the endogenous promoters of its target genes, such as Pdk4 and Mcad, in Zbtb7c-KO MEFs. The $\mathrm{ChIP} /$ re-ChIP assays performed using Pgc1 $\alpha$ and antiacetylated lysine antibodies showed decreased binding of acetylated Pgc- $1 \alpha$ in the Zbtb7c-KO MEFs. The ChIP assays also showed increased acetylation of histones $\mathrm{H} 3$ and $\mathrm{H} 4$ in the endogenous Pgc-1 $\alpha$ target promoters Pdk4 and Mcad because of increased Pgc- $1 \alpha$ binding (Fig. 6c, d). These results showed that $Z \mathrm{btb} 7 \mathrm{c}$ repressed the SIRT1 gene. In the absence of Zbtb7c, lysine residues of Pgc-1 $\alpha$ were deacetylated by derepression of SIRT1, which may explain the inhibition of adipocyte differentiation and fatty acid synthesis and/or increased fatty acid oxidation. These data may explain the apparent decrease in abdominal adipose tissue in Zbtb7c-KO mice (Fig. 1b).

\section{Zbtb7c expression is increased and SIRT1 expression is decreased in mice fed an HFD and aging mice}

Having revealed the molecular mechanism of SIRT1 transcription repression by Zbtb7c and p53, we investigated the physiological significance of our findings. First, we investigated the expression levels of Zbtb7c and SIRT1 in mice fed a normal chow diet and a HFD. Compared to the normal chow-fed mouse controls, the Zbtb7c level was increased by $2-8$-fold and the SIRT1 mRNA level was downregulated by $59-85 \%$ in the HFD-fed mice (Fig. 7a, b. d). Additionally, at the protein level, Zbtb7c expression was increased and SIRT1 expression was downregulated in the eWAT and liver of the HFD-fed mice (Fig. 7c, e).

In addition, we investigated the expression patterns of $Z b t b 7 c$ and Sirt1 during aging. As mice age into adulthood and reach the late adult period, $Z b t b 7 c$ mRNA expression was increased up to 8-80-fold, and Sirt1 was repressed by more than $80 \%$ in both WAT and liver tissues. We observed similar changes in protein expression (Fig. $7 \mathrm{f}-\mathrm{i}$ ). These data suggest that an HFD and aging can cause increased Zbtb7c expression and thereby decreased Sirt1 expression, which can affect diverse cellular processes (e.g., lipid metabolism) controlled by Sirt1 target genes, such as Pgc-1 $\alpha$, Foxo1, Foxo3, p53, Notch, Nf- $k b$, Hif- $1 \alpha$, and Srebp-1c.

\section{Discussion}

SIRT1 has been studied for its role in caloric restriction, prevention of aging-related disease, and maintenance of metabolic homeostasis. Despite numerous publications on SIRT1, our understanding of the molecular mechanism of how the SIRT1 gene is regulated by diet conditions and aging remains limited. Activation of SIRT1 by the activator resveratrol or overexpression of SIRT1 can be beneficial for the treatment of metabolic and neurodegenerative diseases 


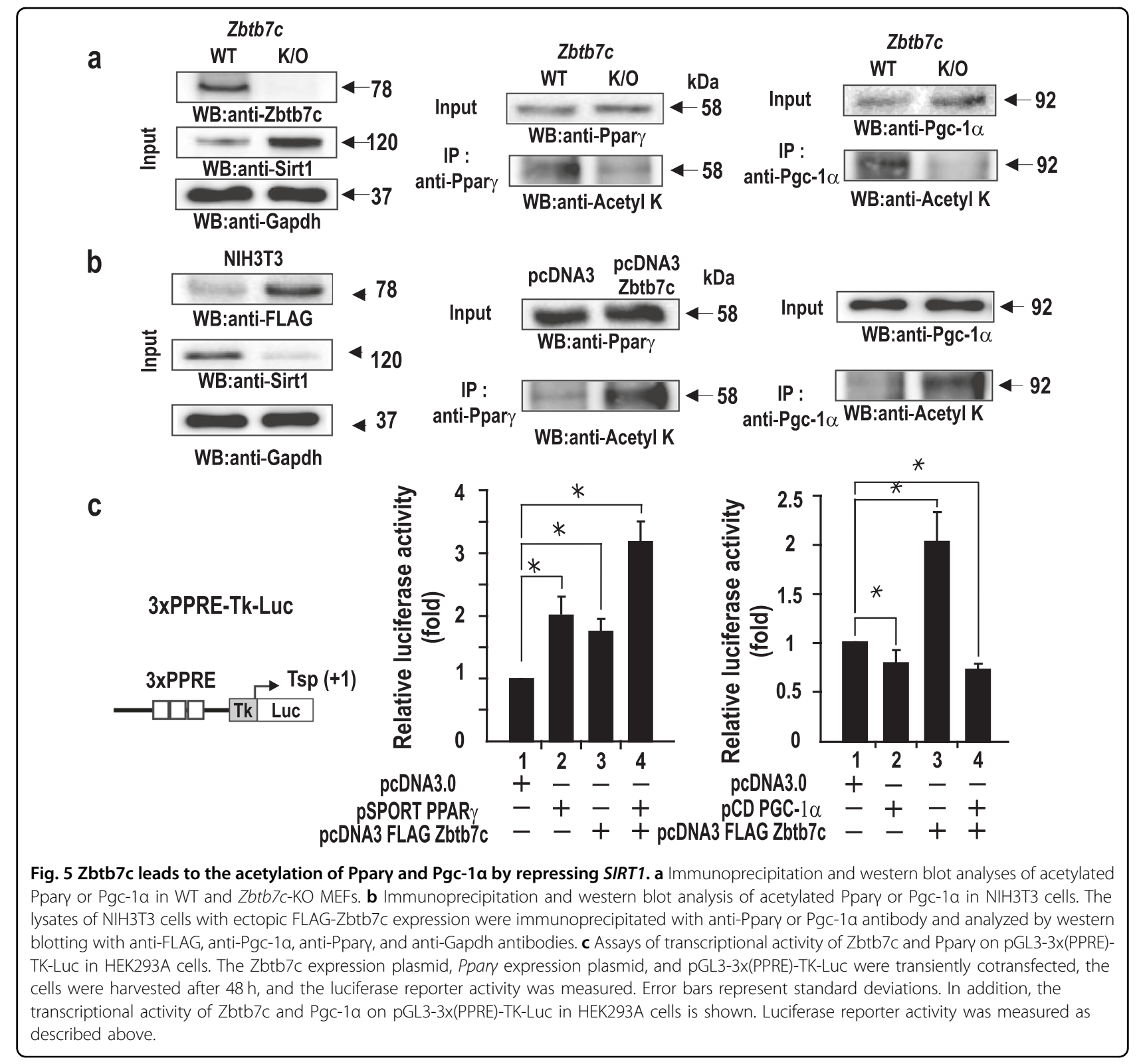

such as type 2 diabetes, obesity, Alzheimer's disease, and Parkinson's disease.

Previously, we and others showed that ZBTB protein family members such as HIC1 and HIC2 regulated transcription of the SIRT1 gene. HIC1 is a transcriptional repressor that interacted with p53 and suppressed agedependent cancer development in mice. The loss of HIC1 function promoted tumorigenesis by derepressing SIRT1 and thereby attenuated p53 function by deacetylation, which increases cancer risk in mammals ${ }^{14}$. Recently, we found that HIC2, a homolog to HIC1 with differing intermediate domains, is a transcriptional activator of SIRT1 and may protect the heart from ischemia/reperfusion (I/R) injury ${ }^{15}$.
Interestingly, the tumor suppressor protein p53 was previously shown to be a major regulator that activated or repressed the SIRT1 gene depending on the physiological conditions. SIRT1, upon DNA damage stress, repressed transcription by binding to the two proximal p53-binding elements ${ }^{14}$. In contrast, upon acute nutrient withdrawal, p53 complexed with Foxo3a bound to the proximal p53binding sites and activated mouse Sirt1, in which both p53RE1 and p53RE2 were equally important ${ }^{5}$. High nucleotide sequence homology of the proximal promoter regions (up to $-220 \mathrm{bp}$ regulatory region $5^{\prime}$-upstream from the ATG translation initiation codon) of human and mouse sirtuin genes suggested the functional importance of p53 elements and molecular events involving p53 and 


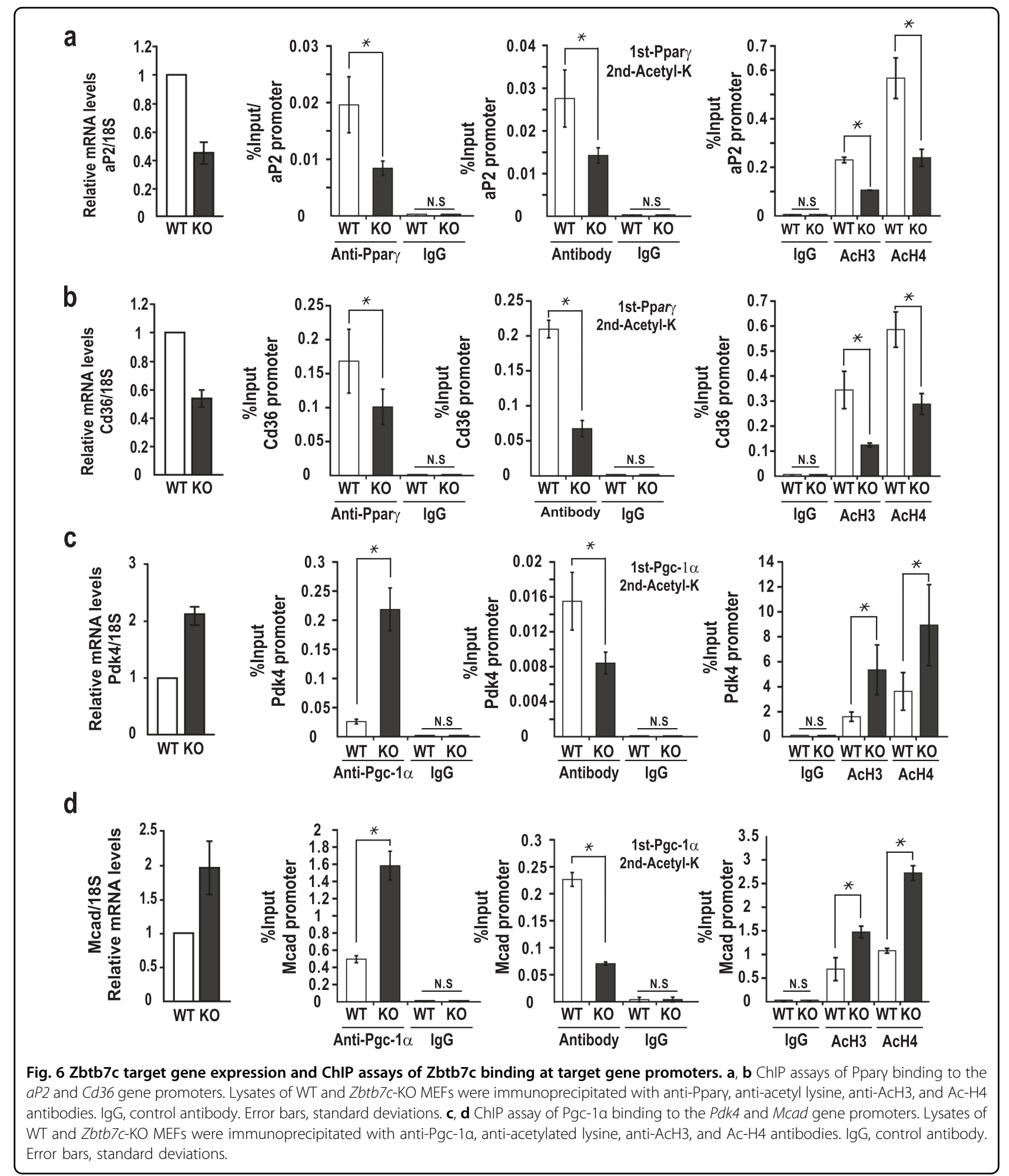

its interacting protein partners, such as Zbtb7c, in SIRT1 gene transcription. Further, $5^{\prime}$-upstream regions beyond the -249 bp of the human SIRT1 and mouse Sirt1 genes (from the ATG translation initiation codon) showed little nucleotide sequence homology. Accordingly, the molecular events revealed at the human SIRT1 proximal promoter region $(-248 \mathrm{bp}$ from the ATG translation codon) may be particularly applicable to the mouse Sirt1 gene, especially under starvation or HFD-fed conditions and in aging mice (Fig. 8). 


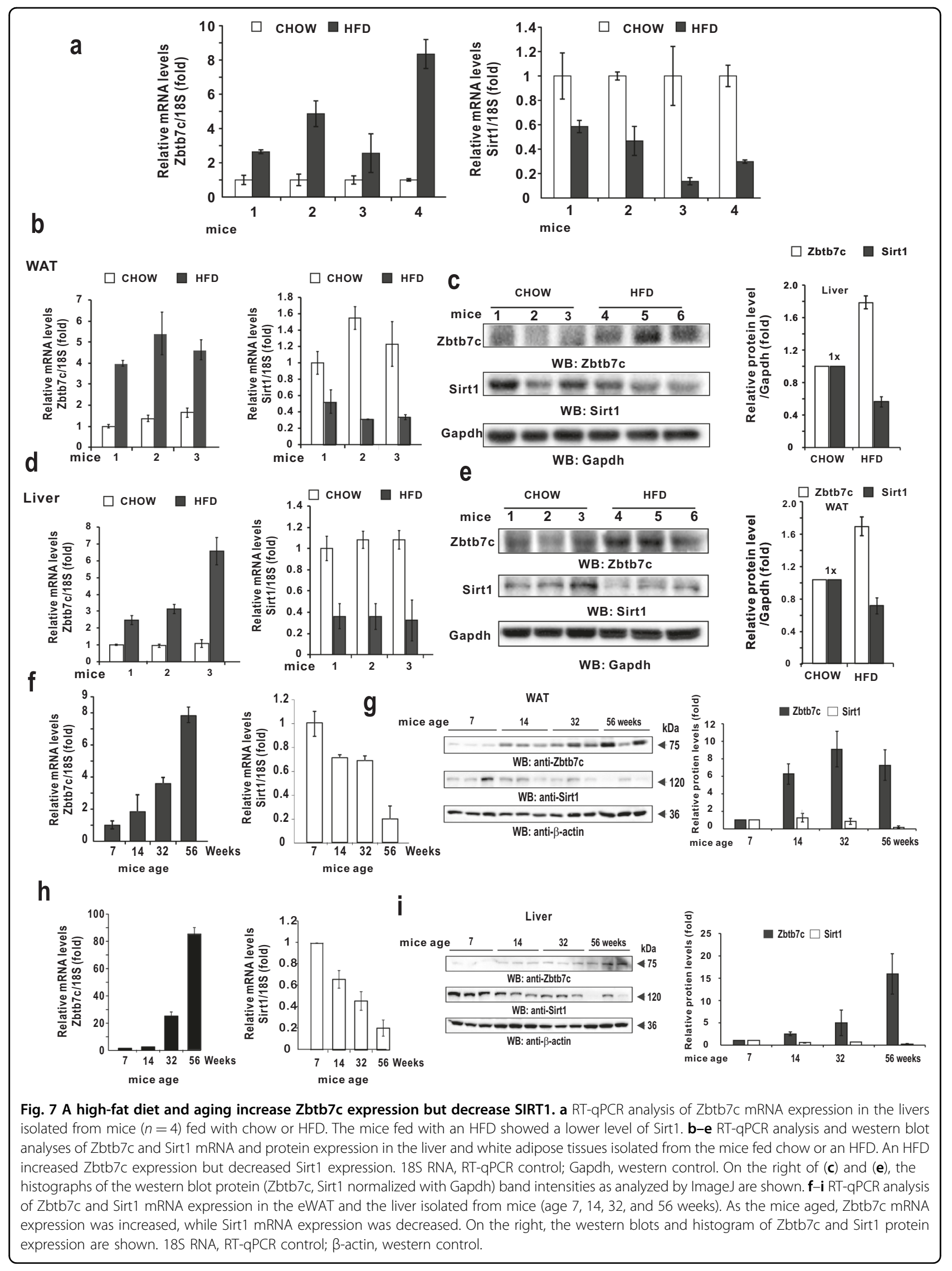




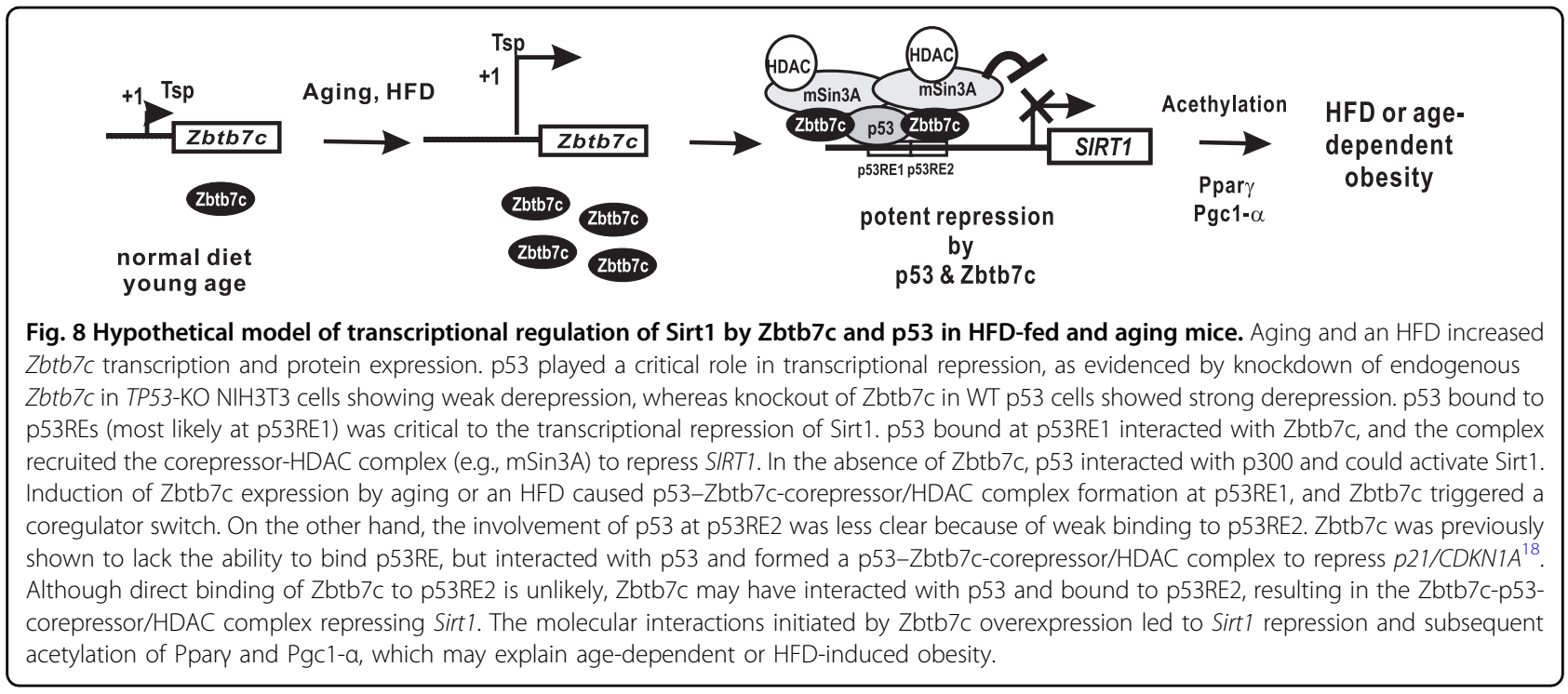

Notably, although the two p53-responsive elements of the mouse Sirt1 have been characterized to be functional in regulating Sirt1 expression, the two mouse p53responsive elements ( -178 and $-168 \mathrm{bp}$ responsive elements both identified through the Genomatrix software package) did not show high sequence similarity to the well-established p53 binding consensus sequence (5'-WWWCGTGDDRRR-3') of p53 target genes regulating cell cycle arrest and apoptosis in the previous studies $^{5,28}$.

In this study, considering the initial observations that (1) abdominal adipose tissues were greatly reduced in old $Z b t b 7 c-\mathrm{KO}$ mice, (2) deletion of the Zbtb7c gene led to significant effects on lipid metabolism, and (3) the negative correlation between SIRT1 and Zbtb7c expression, we investigated and proposed a model of how SIRT1 gene transcription might be regulated in mice fed an HFD and in aging mice. Intriguingly, Zbtb7c induced by an HFD and aging interacted with p53 and represses SIRT1 expression, in which p53RE2 (which is closer to the ATG translation start codon) was more critical. Zbtb7c increased the binding of the corepressor mSin3A-HDAC complex to the p53-Zbtb7c complex at the p53REs, which potently repressed SIRT1 gene transcription (Fig. 8).

Interestingly, in old Zbtb7c KO mice, abdominal adipose tissue mass was significantly reduced. The SIRT1 promoter is thought to contain transcription regulatory elements important in SIRT1 expression and thereby adipocyte differentiation and adipogenesis. Zbtb7c mRNA expression has been shown to be increased by consumption of an HFD and aging. Aged Zbtb7c-KO mice in our study showed a decrease in abdominal fat tissue. Furthermore, microarray analyses of $Z b t b 7 c-\mathrm{KO}$ mouse liver and white adipose tissues revealed significant changes in the expression of genes involved in lipid metabolisms, such as aP2, Fans, Cd36, Fsp27, Cpt1, Mcad, $P d k 4$, and SIRT1. Together, these findings suggested that Zbtb7c is an important regulator of lipid metabolism. Previous studies indicated that SIRT1 directly interacted with and regulated the activity of two major transcription factors and coregulators of lipid metabolism, Ppary, and Pgc- $1 \alpha$, via lysine deacetylation ${ }^{2,26}$ Because Zbtb7c represses SIRT1, it may also affect the expression of target genes such as Ppary and Pgc-1 $\alpha$, as we demonstrated in Zbtb7c-KO MEFs (Fig. 6). Because deacetylation inactivates Ppary but activates Pgc-1 $\alpha$, in our study, the target genes of Ppary were repressed, and the target genes of Pgc- $1 \alpha$ were upregulated by derepressed SIRT1 expression in the absence of Zbtb7c.

Zbtb7c, a potent transcriptional repressor of Sirt1 under HFD feeding and aging conditions, may also affect various physiological processes regulated by Sirt1, such as cell survival, longevity, growth arrest, DNA repair, glucose metabolism, adipogenesis, inflammatory responses, and fatty acid oxidation. The results of our study suggested broad roles and the importance of Zbtb7c as a regulator of physiology. We were able to demonstrate that Zbtb7c is a regulatory protein linking stress, such as that induced by an HFD or aging, with SIRT1 expression. We were able to identify a highly specific molecular target proximal promoter element (p53RE2) that can be engineered to control the expression of SIRT1 in the liver, pancreas, skeletal muscle, adipose tissues, etc., which can be beneficial in glucose and lipid metabolism, metabolic syndrome, pancreatic beta-cell function and insulin secretion, type 2 diabetes, longevity, etc.

\section{Acknowledgements}

This work was supported by Do-Yak Research Grant 2011-0028817 (to M.-W.H.) and Medical Research Center Grant $2011-0030086$ (to M.W.H.) from the National Research Foundation of Korea (NRF) of the Korean Government (MSIP). 
W.-I.C. was supported in part by a research grant (NRF-2018R1D1A1B07047275) from the NRF of the Ministry of Science and ICT of Korea.

\section{Author details}

${ }^{1}$ Brain Korea FOUR Project for Medical Science, Department of Biochemistry \& Molecular Biology, Yonsei University School of Medicine, 50-1 Yonsei-Ro, SeoDaeMoon-Ku, Seoul 03722, Korea. ${ }^{2}$ Graduate School of Medical Science and Engineering, Korea Advanced Institute of Science and Technology, DaeJeon 34141, Korea. ${ }^{3}$ KAIST Institute for the BioCentury, Korea Advanced Institute of Science and Technology, Daejeon 34141, Korea

\section{Author contributions}

W-I.C., B-N.J., J-H.Y., and S.-H.C. performed the research experiments; Hail Kim analyzed the data; M-W.H. designed the research, analyzed the data, and wrote the paper

\section{Conflict of interest}

The author declares no competing interests.

\section{Publisher's note}

Springer Nature remains neutral with regard to jurisdictional claims in published maps and institutional affiliations.

Supplementary information The online version contains supplementary material available at https://doi.org/10.1038/s12276-021-00628-5.

Received: 4 September 2020 Revised: 23 March 2021 Accepted: 29 March 2021.

Published online: 20 May 2021

\section{References}

1. Imai, S., Armstrong, C. M., Kaeberlein, M. \& Guarente, L. Transcriptional silencing and longevity protein Sir2 is a NAD-dependent histone deacetylase. Nature 403, 795-800 (2000).

2. Haigis, M. C. \& Sinclair, D. A. Mammalian sirtuins: biological insights and disease relevance. Annu. Rev. Pathol. 5, 253-295 (2010).

3. Noriega, L. G. et al. (2011) CREB and ChREBP oppositely regulate SIRT1 expression in response to energy availability. EMBO Rep. 12, 1069-1076 (2011)

4. Xiong, S., Salazar, G., Patrushev, N. \& Alexander, R. W. FoxO1 mediates an auto feedback loop regulating SIRT1 expression. J. Biol. Chem. 286, 5289-5299 (2011).

5. Nemoto, S., Fergusson, M. M. \& Finkel, T. Nutrient availability regulates SIRT1 through a forkhead-dependent pathway. Science 306, 2105-2108 (2004).

6. Jin, J., lakova, P., Jiang, Y., Medrano, E. E. \& Timchenko, N. A. (2011) The reduction of SIRT1in livers of old mice leads to impaired body homeostasis and inhibition of liver proliferation. Hepatology 54, 989-998 (2011).

7. Jin, Q. et al. C/EBPalpha regulates SIRT1 expression during adipogenesis. Cell Res. 20, 470-479 (2010).

8. Luo, J. et al. Negative control of p53 bySir2alpha promotes cell survival under stress. Cell 20, 137-148 (2001).

9. Brunet, A. et al. Stress-dependent regulation of FOXO transcription factors by the SIRT1 deacetylase. Science 303, 2011-2015 (2004).
10. Katto, J., Engel, N., Abbas, W., Herbein, G. \& Mahlknecht, U. Transcription factor NFkB regulates the expression of the histone deacetylase SIRT1. Clin. Epigenetics 5, 11 (2013).

11. Zhang, H. N. et al. Involvement of the p65/RelA subunit of NF-kappaB in TNFalpha-induced SIRT1 expression in vascular smooth muscle cells. Biochem. Biophys. Res. Commun. 397, 569-575 (2010).

12. Yeung, F. et al. Modulation of NF-kappaB dependent transcription and cell survival by the SIRT1 deacetylase. EMBO J. 23, 2369-2380 (2004).

13. Pardo, P. S. \& Boriek, A. M. (2012) An autoregulatory loop reverts the mechanosensitive SIRT1 induction by EGR1 in skeletal muscle cells. Aging 4 456-461 (2012).

14. Chen, W. Y. et al. Tumor suppressor HIC1 directly regulates SIRT1 to modulate p53-dependent DNA-damage responses. Cell 123, 437-448 (2005).

15. Song, J. Y. et al. (2019) HIC2, a new transcription activator of SIRT1. FEBS Lett. 593, 1763-1776 (2019).

16. Brunet, A. et al. Stress-dependent regulation of FOXO transcription factors by the SIRT1 deacetylase. Science 303, 2011-2015 (2004).

17. Reuter, S. et al. APM-1, a novel human gene, identified by aberrant cotranscription with papillomavirus oncogenes in a cervical carcinoma cell line, encodes a BTB/POZ-zinc finger protein with growth inhibitory activity. EMBO J. 17, 215-222 (1998).

18. Jeon, B. N. et al. ZBTB7C interacts with p53 and represses its ability to activate transcription of p21WAF1/CDKN1A. Cancer Res. 72, 1137-1148 (2012).

19. Lee, K. M. et al. The proto-oncoprotein ZBTB7C represses transcriptional activation of CDKN1A by MIZ-1 through competitive binding. Oncogene $\mathbf{3 1}$ 1442-1458 (2012)

20. Jeon, B. N. et al. Zbtb7c increases FASN expression by modulating the DNA binding of SREBP-1C and Sp1 at the proximal promoter. J. Lipid Res. 53, 755-766 (2012)

21. Choi, W. I. et al. Zbtb7c is a critical gluconeogenic transcription factor that induces glucose-6-phosphatase and phosphoenolpyruvate carboxykinase 1 genes expression during mice fasting. Biochim. Biophys. Acta Gene Regul. Mech. 1862, 643-656 (2019).

22. Hur, M. W., Yoon, J. H., Kim, M. Y., Ko, H. \& Jeon, B. N. Kr-POK (ZBTB7C) regulates cancer cell proliferation through glutamine metabolism. Biochem. Biophys. Acta Gene Regul. Mech. 1860, 829-838 (2017).

23. Kohn, A. D. et al. Construction and characterization of a conditionally active version of the serine/threonine kinase Akt. J. Biol. Chem. 273, 11937-11943 (1998).

24. Wang, Q. et al. Protein kinase B/Akt participates in GLUT4 translocation by insulin in L6 myoblasts. Mol. Cell Biol. 19, 4008-4018 (1999).

25. Guilherme, A., Virbasius, J. V., Puri, V. \& Czech, M. P. Adipocyte dysfunctions linking obesity to insulin resistance and type 2 diabetes. Nat. Rev. Mol. Cell. Biol. 9, 367-377 (2008).

26. Gerhart-Hines, Z. et al. Metabolic control of muscle mitochondrial function and fatty acid oxidation through SIRT1/PGC-1alpha. EMBO J. 26, 1913-1923 (2007).

27. Qiang, L. et al. Brown remodeling of white adipose tissue by SIRT1-dependent deacetylation of Ppary. Cell 150, 620-632 (2012).

28. Koh, D. I. et al. KAISO, a critical regulator of p53-mediated transcription of CDKN1A and apoptotic genes. Proc. Natl Acad. Sci. USA 111, 15078-15083 (2014). 